Rel at i onshi $p$ anong frequency, temper at ure of the cool ing water and beamcurrent in submi I i met er wave gyr ot r on FU I V

\begin{tabular}{|c|c|}
\hline 著者 & $\begin{array}{l}\text { HORI Toshi hi ro, I DEHARA Toshi taka, SASAGAMA } \\
\text { H royuki, W MRA At sushi, OGAMA I samu, M TSUDO } \\
\text { Sei tar o }\end{array}$ \\
\hline $\begin{array}{l}\text { jour nal or } \\
\text { publ i cat } i \text { on } \mathrm{title}\end{array}$ & Revi ew of Sci entific Instruments \\
\hline vol une & 76 \\
\hline nunber & 2 \\
\hline page $r$ ange & 023502-1-023502- 6 \\
\hline year & $2005-01$ \\
\hline URL & ht t p: //hdl . handl e. net /10098/1605 \\
\hline
\end{tabular}




\title{
Relationship among frequency, temperature of the cooling water and beam current in submillimeter wave gyrotron FU-IV
}

\author{
Toshihiro Hori \\ National Institute of Information and Communications Technology, 4-2-1 Nukui-Kita, Koganei, \\ Tokyo 184.8795, Japan \\ Toshitaka Idehara, Hiroyuki Sasagawa, Atsushi Kimura, \\ Isamu Ogawa, and Seitaro Mitsudo \\ Research Center for Development of Far-Infrared Region, University of Fukui, 3-9-1 Bunkyo, \\ Fukui 910.8507 , Japan
}

\begin{abstract}
The frequency characteristics of the gyrotron FU-IV in free running mode are examined precisely using heterodyne mixing technique. First, the frequency characteristics are measured and the short-term spectral bandwidths and the drifts of the center frequency are found to be less than $10 \mathrm{kHz}$ full width at half maximum and about $10 \mathrm{MHz}$ during 20 min, respectively. Next, an effective water-cooling system reduced the drifts of the center frequency within a range of several $\mathrm{MHz}$. Then, the frequency, the cooling-water temperature, the beam current and the output power are monitored simultaneously to examine the relationship among these parameters. The results indicate that the beam current drift correlate with the frequency drift due to frequency pulling effect as well as the thermal expansion and contraction of the resonant cavity.
\end{abstract}

\section{MTRODUCTION}

In the far infrared (FIR) and submillimeter wave (SMMW) regions, only a few types of radiation sources yield a coherent, continuous-wave (cw) and high output power signals. Gyrotrons are among these sources and have found applications in various fields. ${ }^{1-3}$ The Research Center for Development of Far-Infrared Region at University of Fukui has developed FU Series of millimeter and SMMW gyrotrons (FU-I, FU-II, FU-III, FU-IV, FU-IVA, FU-V, and LOG). Meanwhile, National Institute of Information and Communications Technology (NICT) has developed a system for measuring frequency precisely in SMMW, using heterodyne mixing techniques. In these situations, collaboration work has started on precise frequency measurement of the SMMW gyrotrons.

Various experiments on precise frequency measurements of high-power gyrotrons ${ }^{5-9}$ have been carried out, to know the frequency characteristics and find their dependence on operational parameters. In SMMW gyrotrons also, to know the frequency characteristics and the factors that affect frequency stability is essential for practical applications such as electron spin resonance (ESR) spectroscopy and plasma diagnostics using scattering measurements. Some experimental results have already been obtained and frequency stabilization has been discussed in our previous report. ${ }^{10}$ In the present experiments, we are interested in getting more precise information on the short-term spectral bandwidths and the drifts of the center frequency during operation.

As a first step, we measured the frequency characteris- tics of FU-IV operating in the $300 \mathrm{GHz}$ range in free running mode. The results show that the short-term spectral bandwidths are less than $10 \mathrm{kHz}$ and the drifts of the center frequency, in other words, the frequency accuracy, are about $10 \mathrm{MHz}$ in $20 \mathrm{~min}{ }^{11}$ The short-term spectral bandwidths are as narrow as the reported data for optically pumped FIR lasers, that is, the spectral bandwidth of $5 \mathrm{kHz}$ and the accuracy of $500 \mathrm{kHz}^{12}$

The results of our experiments indicate that the ratios of the drift frequency to the center frequency are on the order of $1 \times 10^{-5}$. One reason for the frequency drift is obviously the thermal expansion and contraction of the resonant cavity of the gyrotron. Reference 13 shows that the copper cavity has an expansion coefficient equal to $1.67 \times 10^{-5} / \mathrm{deg}$. To study the role which the thermal expansion and contraction plays on the frequency characteristics, we measured the temperature of the cooling water as well as the frequency and found good correlation between them. Through efficient control, the drifts of the center frequency were reduced to several MHz. ${ }^{11}$

In an attempt to understand in more details the factors that cause the drifts of the frequency, we simultaneously monitored the beam current, the output power, the coolingwater temperature and the frequency. As a result, the relationship among the frequency, the cooling-water temperature, and the beam current are found. ${ }^{11}$ In this article we present and discuss the results of our experiments. The data indicate the influence of the frequency pulling effect of the beam current on the frequency characteristics. 


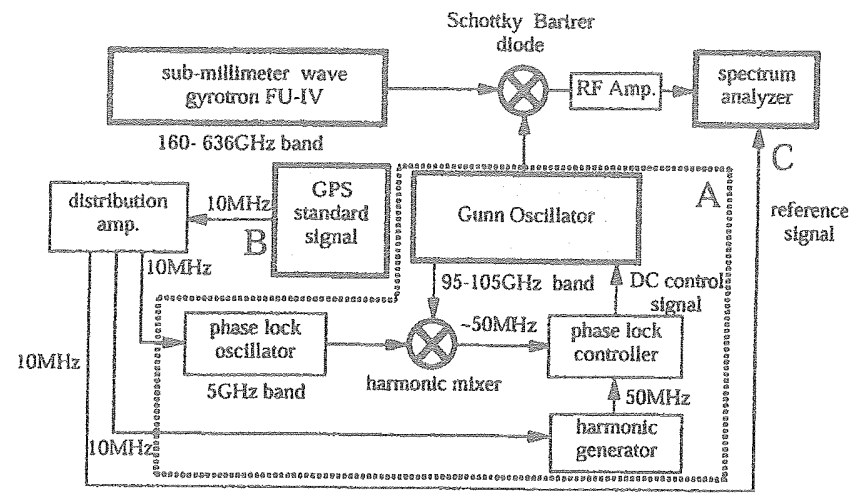

FIG. 1. Experimental setup of frequency measurement system for gyrotron FU.IV.

\section{PRECISE RREQUENCY MEASUREMENT USING METERODVNE TECHNOUES}

\section{A. Expermental setu}

To measure the frequency characteristics of the gyrotron radiation, heterodyne hamonic mixing lechnique is used. The frequency measurement system used in these experiments was initially developed to measure the frequency of optically pumped FIR lasers. This system is made up of a point-conact Schotky barrier diode (SBD) mixer, a phaselocked $100 \mathrm{GH}$ band Gunn oscillator and global positioning system (GPS)-synthesized $10 \mathrm{MHz}$ standard signal distributing facilly. The layout of the apparatus is shown in Fig. 1 and the main components are briefly described below.

\section{Gyrotron}

FU-IV is operated at the frequency of $300-302 \mathrm{GHz}$ with a maximum output power of about $20 \mathrm{~W}$. The radiated wave is transmitted through a circular waveguide and divided into two beams. One goes to the power-meter Scientech. Model 362 while the other goes into the SBD mixer of the heterodyne system. To measure the total power, the absolute calorimetric method with a water load is used. The output power is sufficient to produce an appropriate signal in the SBD mixer.

\section{2. $\$ 90$ hererodyne harmonic mixer}

An IT7 chip from the University of Virginia is used as a frequency mixer. The intermediate frequency, $f_{I F}$, is given by Eq. (1):

$$
f_{I F}=f_{\text {gyro }}-3 \times f_{\text {gunn }}
$$

where $f_{\text {gyro }}$ and $f_{\text {guna }}$ are the frequencies of the gyrotron and the phase-locked Gunn oscillator, respectively. The maximum operating current of the SBD is about $300 \mu \mathrm{A}$. The IF signals generated in the SBD mixer are amplified with lownoise amplifiers (Trontech. W1G2H, NF=1.2 dB, 30 dB *2) and the signal is displayed on a spectrum analyzer.

\section{Phase-locked Cum oseillator}

A commercial Gunn diode to which a phase locking loop (PLL) is added is used as a local oscillator. Its configuration is shown in Fig. 1A. In the frequency range from 95 to $105 \mathrm{GHz}$ the output level is over $5 \mathrm{~mW}$. A reference signal $(10 \mathrm{MHz})$ is generared from a GPS-synthesized signal generator, Symmetricom. $58540 \mathrm{~A}$ (Fig. 1B). The results show that the frequency stability is within a range of $1 \mathrm{~Hz}$ at $100 \mathrm{GHz}$ and $10 \mathrm{~Hz}$ at $1 \mathrm{THz}$. Synthesized $5 \mathrm{GHz}$ range signals are generated from a phase-locked oscillator (PLO). The frequency difference between the Gun oscillator and the 20th harmonics of the PLO output is used for phase-locking the Gunn oscillator.

\section{Distribution of GPS-5ynthesized $10 \mathrm{MH}$ srandard signals}

The $10 \mathrm{MHz}$ signal generated by the GPS-synthesized signal source is distributed to all components of the frequency measurement sysiem using a distribution amplifier (HP5087A).
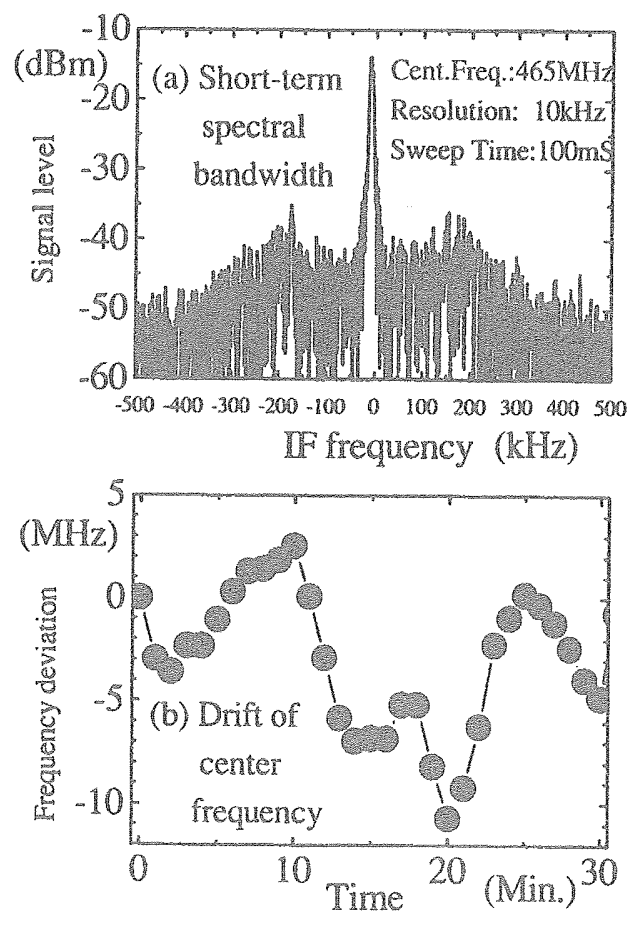

FIG. 2. Results of the measurement of the frequency when the coolingwater temperature is not controlled 

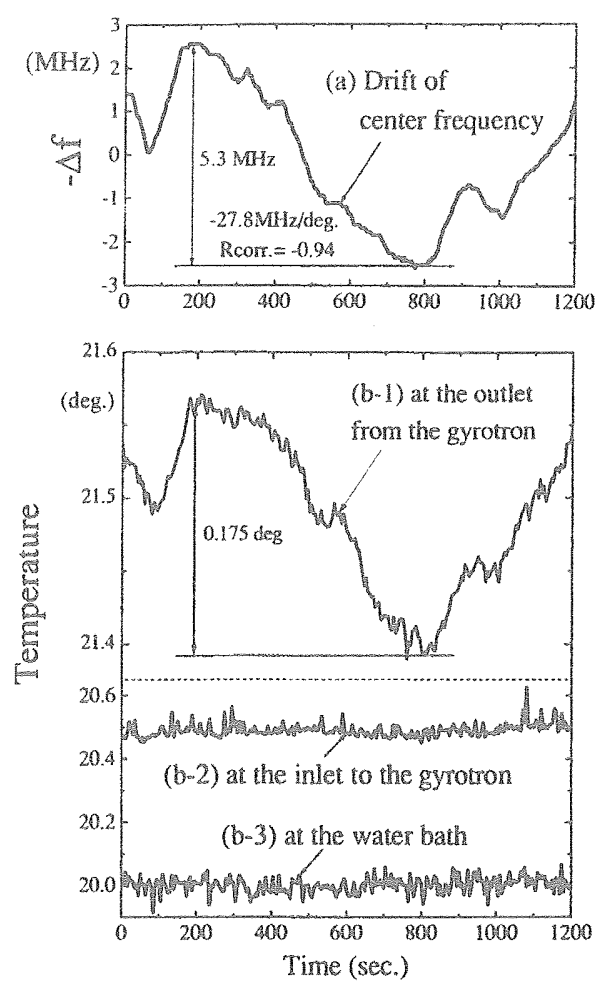

FIG. 3. Results for measurement of cooling-water temperature at three points and the frequency.

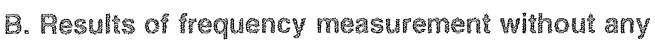 control}

Typical examples of results obtained withoul a coolingwater control are shown in Figs. 2(a) and 2(b).

\section{Shoreferm spectrat bandwidth}

Figure 2(a) shows the results obtained for the short-term spectral bandwidths. As can be seen, the spectral bandwidth is $10 \mathrm{kHz}$ (FWHM) at maximum. The limitation on the measurements of spectral bandwidhs, that is, $10 \mathrm{kHz}$ (FWHM), is not due to the resolution of the gyrotron spectrum itsel, but to the drifts of the center frequency. The drifts of the center frequency do not allow the frequency to be measured at a higher resolution. Therefore, the actual spectral bandwidth is probably less than $10 \mathrm{kHz}$ (FWHM). The estimated value is as small as the nominal values obtained for optically pumped FIR lasers, that is, $5 \mathrm{kHz}{ }^{12}$ It must be noted that the two side peaks that appear at a separation of about $200 \mathrm{kHz}$ from the center frequency in Fig. 2(a) come from the spectrum of the phase-locked Gunn oscillator.

\section{Drifs of center irequency (accuracy of frequency)}

Figure 2(b) illustrates the drifts of the center frequency, that is, the accuracy of the frequency. The experimental results show that the frequency drifts are about $10 \mathrm{MHz}$ in $20 \mathrm{~min}$.

\section{Aesults obrained by simulaneous monichoring of gyrotron parameters}

FU-IV is operated at $300-302 \mathrm{GHz}$ and the drifts of the center frequency are about $10 \mathrm{MHz}$. Thus, the relative drifts of frequency are in the order of $1 \times 10^{-5}$. As already mentioned, we consider that thermal expansion and contraction of the resonant cavity is one of the factors responsible for the drifts of the requency. Therefore, the cooling-water system was improved to reduce the frequency drifts. In addition, the electron beam current and the output power of the gyrotron are also monitored simultaneously with the frequency and cooling-water temperature to examine the relationship among these parameters. The experimental results are shown below.

\section{Insuluence of coohng-uarer semperature on hequengy}

A water chiller (NesLab. Corp. RTE-210) was added to stabilize the temperature of the cooling water. In the experiments, three thermocouples with voltage-corrected ICs (Analogue Devices, AD595) are used to measure the temperature in both the startup phase and cw operation phase at three different points; in the water buk, at the inlet to the gyrotron, and also at the outlet. Obtained data are processed using a digital multimeter and a personal computer with a GPIB interface.

The results for cw operation are shown in Figs. 3(a), $3(b-1), 3(b-2)$, and $3(b-3)$. The drifts of the temperature at the outlet of the gyrotron, which are shown in Fig. $3(\mathrm{~b}-1)$, are well correlated with the frequency drifts presented in Fig. $3(a)$, while the temperatures at the inlet to the gyrotron [Fig.
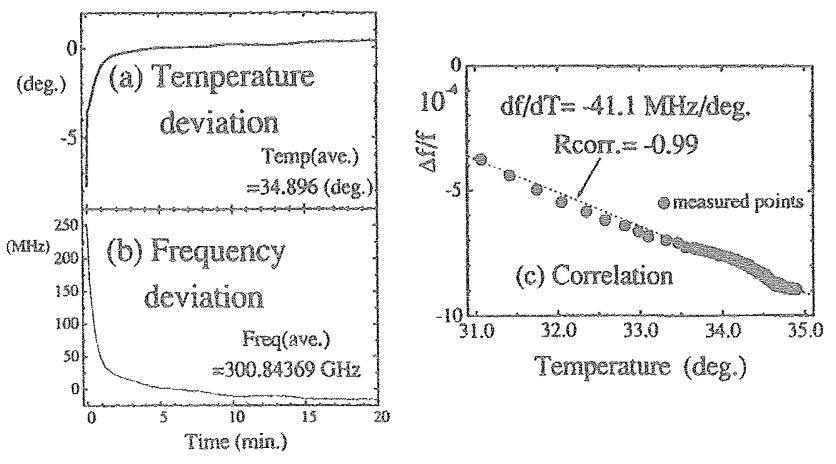

FIG. 4. Relationship between the cooling-water ternperature and the frequency in startup phase. 

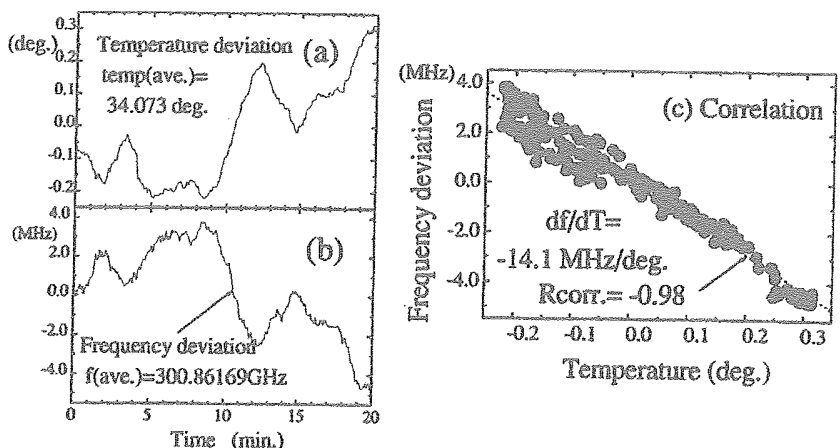

FIG. 5. Relationship between the cooling-water temperature and the frequency in cw operation phase. 3(b-2)] and in the water tank [Fig. 3(b-3)] are not correlated. This result shows that the thermal expansion and contraction of the cavity is related to the frequency drifts. It must be noted that the frequency decreases when the temperature rises.

The data for the startup phase are shown in Figs. 4(a)4(c). In Fig. 4(a), the temperature rise was about $8^{\circ}$ and frequency decrease was about $270 \mathrm{MH}$. The correlation between the temperature and frequency is shown in Fig. 4 (c). The results demonstrate a strong correlation with a correlation coefficient of about 0.99 .

Data for the drifts of the temperature and the frequency in cw operation phase are shown in Figs. $5(a)$ and $5(b)$. The correlation between the temperature and the frequency is itlustrated in Fig. $5(\mathrm{c})$ and a strong correlation with a coeffcient of about 0.98 is also found.

Next, the results obtained with and without the cooling facility are compared in Figs. 6(a) and 6(b), showing that the frequency is more stable when a temperature-controlled chiller is used. These results indicate the importance of controling cooling-water temperature.

The linear coefficients between the frequency and the temperature and their correlation coefficients are summarized in Table. I with the data from Figs. 3(a), 4(a), 5(a), 6(a), and $6(b)$. The data are arranged according to the peak-to-peak frequency values. As a result, Figs. 4(a), 6(a), 5(a), 3(a), and 6 (b) form a sequence.

\section{2. mintunce of bean curren on wrequency}

When the temperature of the cooling water is stabilized, the drifts of the center frequency remained within a range of

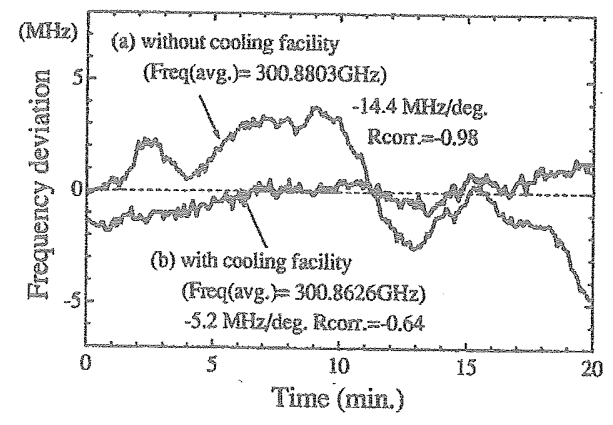

FIC. 6. Stabilizing effect of cooling-water temperature. a few MHz. To examine other factors that may affect the frequency characteristics, the beam current, the output power, the temperature of the cooling water and the frequency are monitored simultaneously. The obtained data, which correspond to an output power of $5 \mathrm{~W}$, are presented in Figs. 7(a)-7(d). Figure 7(d)(d) shows that in this case, frequency drifts are within a range of $1 \mathrm{MHz}$. The strong correlation among the four curves indicate that the beam current is the principal factor for the frequency drifts at least via thermal expansion and contraction of the cavity.

Using the data presented in Figs. 7(a)-7(d) the correlation coefficients are calculated. The correlations between the beam current, the output power, the temperaure of the cooling water and the frequency are shown in Figs. $8(a)-8(0)$. The correlation coefficient between the beam current and the output power is found to be about 0.95 while the correlations between the beam current and frequency and between the output power and the frequency are $0.76[\mathrm{Fig} .8(\mathrm{c})]$ and 0.71 [Fig. 8(e)], respectively. The linear coefficient between the frequency and the cooling-water temperature is $1.5 \mathrm{MHz} / \mathrm{deg}$ with a correlation coefficient of 0.76 [Fig. $8(0)$. These values are listed in Table 1 for discussions below.

\section{DISCUSSION}

We discuss three aspects of the experimental results. The first one is the cause of the slow drifts of the beam current and the water temperature. The second is the time constant of the decrease in frequency in the startup phase and the last is the linear coefficients between the frequency and the cooling-water temperature and their correlation coefficients.

As shown in Fig. 7 , the frequency drifts are related to drifts of the bean current and the water temperature. The

TABLE I. Relationships between the temperature and the frequency.

\begin{tabular}{cccc}
\hline $\begin{array}{c}\text { Figure } \\
\text { No. }\end{array}$ & $\begin{array}{c}\text { Frequency } \\
\text { (peak-to-peak) } \\
\left(M H z_{p-p}\right)\end{array}$ & $\begin{array}{c}\text { Linear } \\
\text { coefficients } \\
(\text { MHz/deg) }\end{array}$ & $\begin{array}{c}\text { Absolute value } \\
\text { of correlation } \\
\text { cosfficient }\end{array}$ \\
\hline $4(a)$ & $\approx 270$ & -41.1 & 0.99 \\
$6(a)$ & 8.6 & -14.4 & 0.98 \\
$5(a)$ & 8.6 & -14.1 & 0.98 \\
$3(a)$ & 5.3 & -27.8 & 0.94 \\
$6(b)$ & 3.3 & -5.2 & 0.64 \\
$8(0)$ & 1.3 & -1.5 & 0.76 \\
\hline
\end{tabular}


problem gives rise to the latter drifts. Possible factors relating to beam current include the drift of heater temperature of thermal electron emission and the drift of acceleration voltage. A possible factor relating to the water temperature is the maximum temperature stability that the chiller can maintain. The maximum temperature stability of the water chiller we used is $0.1 \mathrm{deg}$. The peak-to peak value of water temperature in Fig. 7(c) is about 0.7 deg so about $15 \%$ of the drift can be attibuted to the achievable maximum temperature stability. In future work, to find the causes of the drift of the bean current and the water temperature is very important to our aim of achieving frequency stabilization.

Next, the time constants of the frequency decrease are discussed. Figures $4(a)$ and $4(b)$ show that the time constants of the temperature and the frequency deviations in startup phase are both in the range of $100 \mathrm{~s}$. In contrast, the time constants reported in previous studies are in the range of $0.1 \mathrm{~s}$ in Ref. 6 and of $1 \mathrm{~s}$ in Ref. 7. Below, we discuss the reasons for the large discrepancy between our data and the reported data.

In the case of thermal transfer in one-dimensional slab and wo-dimensional axial configurations, Fourier number is considered as a characteristic time figure that can be used to nomalize the problems. Fourier number is defined as a square of the characteristic length, such as, the distance beiween the heat source and the observation point $L_{0}$, divided by the thermal diffusivity $\alpha_{s}^{13}$ i.e., $L_{0}^{2} / \alpha$. In other words, Fourier number expresses the time constant of the thermal transfer phenomena.

FU-IV is designed for high-frequency, cw mode operation. The inner surface of the cavity is heated by the skin effect. And the outer surface is in direct contact with the cooling water. The inner and outer radii of the cavity are about 1.5 and $10 \mathrm{~mm}$, respectively. Thus, the $L_{0}$ is about $8.5 \mathrm{~mm}$. In contrast, in gyrotrons designed for high-power pulse mode operation, the inner and outer radii are, for ex-
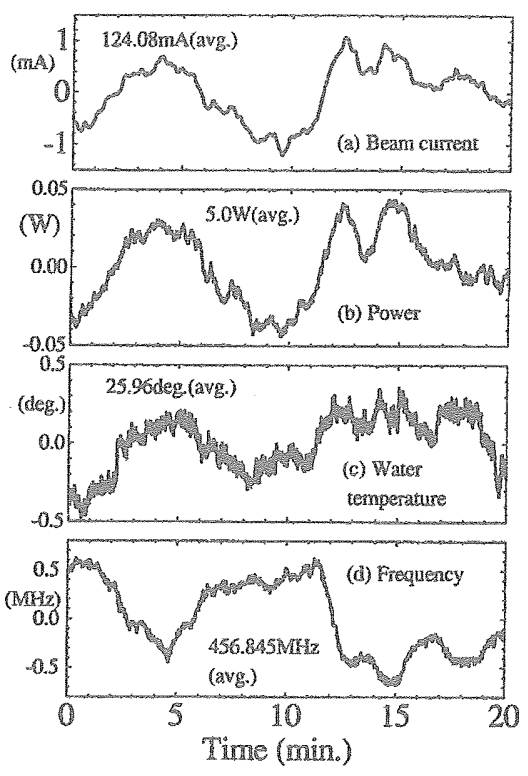

FIG. 7. Results of the simultaneous measurement of the beam current, the output level, the cooling- water temperature and the frequency.

ample, 10 and $12.5 \mathrm{~mm}$, respectively, though the values depend on the situations. The $L_{0}$ is about $2.5 \mathrm{~mm}$. When Fourier number for these two cases is compared, it is more than one-order larger in FU-IV than in other high-power gyrotrons. We think this is the major cause of the discrepancy in the time constants. In addition, when the frequency characteristics are measured during pulse operation, the oscillation is sometimes cutoff before there is sufficient decay in frequency. This will make the measured time constants shorter than expected.
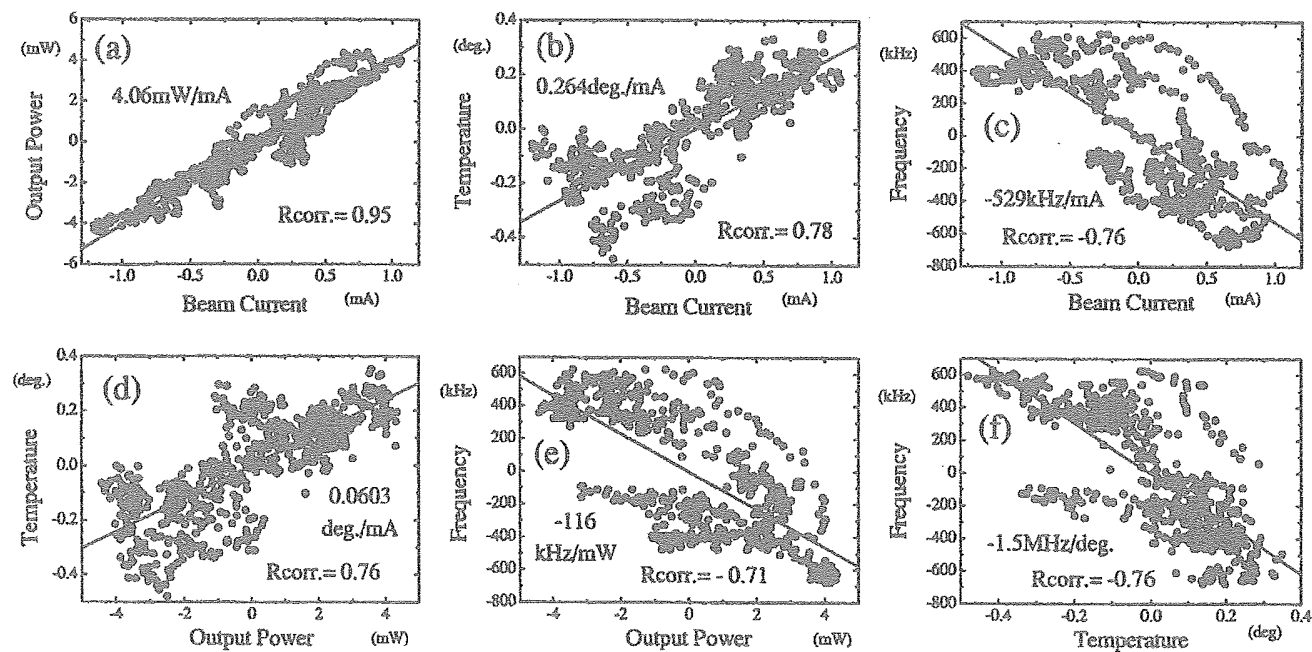

FIG. 8. Correlation coefficients between parameters in Figs. 7(a)-7(d): (a) beam current and output power; (b) beam current and cooling-water temperature; (c) beam cument and frequency; (d) output power and cooling-water temperature; (e) output power and frequency; and (f) cooling-water temperature and frequency. 
We now discuss the linear coefficients between the fre quency and the cooling-water temperaure and their correlation coefficients. Our estimate shows that if the drifts of the frequency are caused by thermal expansion and contraction of the cavity and the temperature is measured at the inner radius of the cavity, the coefficient must be $5.10 \mathrm{MHz} / \mathrm{deg}$ as shown in Eq. (2):

$$
\left(3.00 \times 10^{8}\right) \times\left(1.67 \times 10^{-5}\right)=5.10(\mathrm{MH} 2 / \mathrm{deg}) .
$$

The value obtained in Fig. $8(9)$, of $1.5 \mathrm{MHz} / \mathrm{deg}$ is less than $5.10 \mathrm{MHz} / \mathrm{deg}$ indicating that thermal effect may not be the only cause of the frequency drifts.

When the relationship between the temperature and frequency is examined using the data in Table I it appears that there is a tendency for both the linear coefficients and the absolute values of correlation coefficients to be smaller when the peak-to-peak frequency drifts are smaller, although there are some exceptions, such as, the linear coefficients for Fig. 3(a) and the correlation coefficients for Figs. $6(\mathrm{~b})$ and $8(\mathrm{f})$ We think that these results suggest that another physical mechanism affects the irequency characteristics as well as the thermal effect.

The frequency pulling effect of the beam current in highfrequency gyrotrons has been discussed theoretically. ${ }^{14-16}$ In Ref. 16, it is found that when the magnetic field, the frequency and the beam current and the loaded Q factor of the cavicy are $6.725 \mathrm{~T}, 182.993 \mathrm{GHz}, 120 \mathrm{~mA}$, and 4000 , respectively, the frequency and the beam current are related by a factor of $0.24 \mathrm{MHz} / \mathrm{mA}$. In our case, the frequency tunability is about $50 \mathrm{MHz}$ at $300 \mathrm{GHz}$ in experiments and is considered to be determined roughly by the loaded $Q$ factor of the cavity, that is, about 6000 . As a result, it is similar to the value in Ref. 16. The linear coefficient between the temperature and the beam current is $0.264 \mathrm{deg} / \mathrm{mA}$ [Fig. 8(b)]. If a linear relationship is assumed for 183 and $300 \mathrm{GHz}$, the linear coefficient for $300 \mathrm{GHz}$ is estimated as shown in Eq. (3):

$$
0.24 \times \frac{1}{0.264} \times \frac{300}{183}=1.48(\mathrm{MHz} / \mathrm{deg}) .
$$

This value is roughly in the same range as the linear coefficients between the frequency and the cooling-water temperature and can explain why the linear coefficient between the frequency and the cooling-water temperature is reduced to $1.5 \mathrm{MH} / \mathrm{deg}$ and the correlation coefficient is only about 0.76 [Fig. 8(i)].

Based on our experimental results, we think that various physical mechanisms infuence the frequency characteristics as follows. The drifts of frequency are caused by the thermal effect via cavity expansion and contraction and by the nonthemal frequency pulling effect of the beam current. The time constant of the drifts are in the range of $10 \mathrm{~min}$. The sum of these two effects can be observed in practical measurements. The thermal effect is caused by both the drift of cooling-water temperature and the drift of output power originaing from the beam current drift. The thermal drift is in a range of $10 \mathrm{MHz}$ and when the beam current increases, the freguency decreases. The nonthermal frequency pulling effect of the beam current is around $1 \mathrm{MHz}$ and when the beam current increases, the frequency increases. This nonthemal effect is sometimes hidden by the thermal effect. We think that the above description explains the physical mechanisms that occur in submillimeter wave gyrotron FU-YV.

\section{ACRNOWHEDGMENS}

The authors express their appreciation to the anonymous referee for his valuable suggestion to interpret the experimenally obtained results. This work was partially supported by the Grant-in-Aid from Japan Society for the Promotion of Science.

'J. L. Hirshield, Infrared and Millimeter Woves, edited by K. Buton (Academic, New York, 1979), Vol. 1, p. 1.

${ }^{2} \mathrm{~T}$. Idehara, $\mathrm{T}$. Tatsukawa, 1. Ogawa, Y. Shimizu, S. Makino, and T. Kanemaki, Phys. Fluids B 5, 1377 (1993).

${ }^{3}$ K. D. Hong, G. F. Brand, and T. Idehara, J. Appl. Phys. 74, 5250 (1993) ${ }^{4}$ T. Hori and N. Hiromoto, Jpn. 3 . Appl. Phys., Pan 132,5552 (1993).

${ }^{5} 1$. Rebutf, Technical Digest of the $11 \mathrm{th}$ International Conference IR and MMW, Trrenia, Pisa, llaly, 1986, p. 134

${ }^{6}$ K. Felch, T. S. Chu, J. Feinsiein, H. Huey, H. Jory, J. Neilson, and R. Schumacher, Technical Digest of the 17 th memational Conference $\mathrm{IR}$ and MMW, Pasadena, CA, 1992, p. 184.

G. Dammerz, O Braz, C. T. Iarou, M Kuntze, A Moebius, B Piosczyk and M. Thumm, Technical Digest of the 20th International Conference IR and MMW, Lake Buena Vista, FL, 1995, p. 285.

${ }^{8}$ Y. Terumichi, S. Kubo, A. Ando, Y. Yanagimoto, K. Ogura, H. Tanaka, J Takahashi, I. Tokai, M. Nakamura, T Maekawa, S. Tanaka, and T Ide hara, Technical Digest of the 9 th International Conference IR and MMW, Takarazuka, Hyogo, Japan, 1984, p. 411.

${ }^{9}$ T. Idehara, T. Tatsukawa, H. Tanabe, S. Matsumoto, K. Kunieda, K Hemmi, and T. Kanemaki, Phys. Fivids B 3,1766 (1991).

${ }^{10}$ I. Ogawa, T. Idehara, M. Ui, S. Mitsudo, and W. Foerster, Fusion Eng. Des. 53,571 (2001)

${ }^{11}$ T. Hori, H. Sasagawa, A. Kimura, I. Ogawa, \$. Mitsudo, and T. Idehara Technical Digest of the $28 \mathrm{th}$ Intervational Conference IR and MMW, Ohtsu, Japan, 2003, p. 413

${ }^{12}$ L. R. Zink, P. DeNatale, F. S. Pavone, M. Prevedelli, K. M. Evenson, and M. Inguscio, J. Mol. Spectrosc. 143, 304 (1990).

${ }^{13}$ American Institute of Physics Handbook, 3rd ed., edited by D. E. Gray (McGraw-Hill, New York, 1972).

${ }^{14}$ I. Y. Choe and S. Akn, IEGE Trans. Electron Devices ED-28, 94 (1981).

${ }^{15}$ G. F. Brand, Int. I. IR MMW 4, 247 (1983).

${ }^{16}$ G. F. Brand, Int. J. IR MMW 4, 919 (1983). 